\title{
Adaptive simulations of viscous detonations initiated by a hot jet using a high-order hybrid WENO-CD scheme
}

\author{
Xiaodong $\mathrm{Cai}^{1}$, Ralf Deiterding ${ }^{2}$, Jianhan Liang ${ }^{1 *}$, Yasser Mahmoudi ${ }^{3}$ \\ ${ }^{1}$ Science and Technology on Scramjet Laboratory \\ National University of Defense Technology, Changsha, 410073, China \\ ${ }^{2}$ Aerodynamics and Flight Mechanics Research Group, University of Southampton \\ Highfield Campus, Southampton SO171BJ, United Kingdom \\ ${ }^{3}$ Department of Engineering, University of Cambridge \\ Trumpington Street, Cambridge CB21PZ, United Kingdom \\ *Corresponding author: jhleon@vip.sina.com
}

Colloquium: DETONATIONS, EXPLOSIONS and SUPERSONIC COMBUSTION

Total length: 7040 words $($ Method 1) $=3976($ main text $)+699($ references $)+164($ Fig.1) +229 (Fig.2)

+243 (Fig.3) +247 (Fig.4) +558 (Fig.5) + 558 (Fig.6) +275 (Fig. .7) + 91 (Table.1)

Affirmation: The authors confirm to pay color reproduction charges if applicable. 


\section{Abstract:}

In the present work a sixth-order hybrid WENO-Centered Difference (CD) scheme with an adaptive mesh refinement method is employed to investigate gaseous detonation by injecting a hot jet into a hydrogen-oxygen combustible mixture flowing at supersonic speed. Two-dimensional reactive NavierStokes (NS) equations with one-step two-species chemistry model are solved numerically. The comparison between viscous and inviscid detonation structures shows that due to the absence of both the physical viscosity in Euler equations and minimization of numerical dissipation in the hybrid WENO-CD scheme, very small-scale vortices can be observed behind the detonation front. The diffusion effect in the NS equations suppresses the small-scale vortices, but it has negligible influence on the large-scale vortices generated by Richtmyer-Meshkov (RM) instability and those along the highly unstable shear layers induced by Kelvin-Helmholtz (KH) instability. When studying the same setup in an expanding channel and beyond the point of detonation initiation, it is found that because of the diffusion effect of detached shear layers, any unburned jet flow is consumed quickly and then additional energy is released periodically. Because of the formation of multiple secondary triple points and subsequent shear layers after the shutdown of the hot jet, a highly turbulent flow is produced behind the detonation front. Rather than the commonly known RM instability, the large-scale vortices involved in the highly unstable shear layers dominate the formation of the turbulent flow and the rapid turbulent mixing between the unburned jet flow and burned product. It is found that the size of unburned jets and vortices due to KH instability is growing for larger expansion angles. The further generated turbulent flow resulting from larger sized vortices, significantly enhances the mixing rate behind the Mach stem, leading to rapid consumption of the unburned reactants. Therefore, detonations propagate faster in channels with larger expansion angle and higher expansion ratio.

Key words: viscous detonation; hybrid scheme; diffusion effect; expansion channel; adaptive mesh 
refinement

\section{Introduction}

Due to the superior performance at high Mach numbers, scramjets have become one of the first choices for hypersonic air-breathing propulsion systems. Scramjet combustors adopt the Brayton cycle, whose thermodynamic efficiency is far below than that of detonation combustion [1]. The inherent theoretical advantage of detonation combustion over deflagrative combustion indicates that the performance of scramjets might be improved significantly if detonation-driven reaction can be realized inside the combustor.

Reliable detonation initiation has always been one of the key issues for detonation combustion. While direct initiation [2] can realize initiation quickly but is not generally applicable, an alternative approach for the initiation is to use a hot jet [3]. In [4] detonation initiation was realized successfully in experiments by transmitting a strong hot jet into high speed combustible mixtures with Mach numbers 0.9 and 1.2. In $[5,6]$ detonation initiation and deflagration to detonation transition (DDT) were both conducted experimentally using a hot jet in supersonic combustible mixtures, whereas detonations were initiated accurately through shocks or shock reflections [7-9] induced by the hot jet. We have conducted a series of numerical investigations [10-14] on detonation initiation and propagation in straight channels using a hot jet in supersonic combustible mixtures, where the opensource program AMROC [15-17] (Adaptive Mesh Refinement Object-oriented C++) based on an SAMR (Structured Adaptive Mesh Refinement) framework [18] is utilized. These numerical simulations solve the inviscid reactive Euler equations and apply a classical second-order accurate MUSCL-TVD (Monotone Upstream-centered Schemes for Conservation Laws-Total Variation Diminishing) scheme. 
Compared with the inviscid Euler equations, solving the reactive NS equations in detonation simulations has become an interesting topic of current research [19-23]. However, the typically adopted shock-capturing methods introduce excessive numerical dissipation, which can pollute the diffusive part of the equations. Conversely, the methods which can capture accurately the diffusive terms without numerical dissipation usually tend to lack robustness and stability for discontinuities because of a lack of shock-capturing dissipation.

In the present work, for detonation simulations in supersonic combustible mixtures we solve the reactive NS equations with a simple reaction model by utilizing a robust high-order hybrid WENOCD (Weighted Essentially Non-Oscillatory-Centered Difference) scheme [24, 25] together with the SAMR framework [18]. The overall approach combines the robustness of WENO for discontinuity capturing with the benefit of a centered scheme with low numerical dissipation in smooth solution regions and the efficiency of SAMR. The method has been validated meticulously for Euler as well as NS equations for the simple one-step two-species chemistry and detailed chemistry in [17]. Considering that the actual scramjet combustors are usually expanding for thrust propulsion, understanding the behavior of detonation in expanding channels is important for detonation physics and practical applications. Here we study viscous simulations of detonation combustions in supersonic combustible mixtures in expanding channels. This work is part of an ongoing research program, aiming at providing information to improve the overall understanding of detonation combustion in supersonic combustible mixtures. The remainder of this paper is organized as follows: the computational model is presented in Section 2. Results of detonation simulations are discussed in Section 3, in which the role of diffusion resulting from hydrodynamic instabilities in the propagation mechanism of hydrogen detonations is studied by comparing Euler and NS solutions. Applications of detonations in expanding channels are shown in Section 4, in which detonation propagation during the hot jet injection and 
particularly after the jet shutdown are discussed using NS equations on the development of hydrodynamic instabilities and consequently on the propagation of the detonation wave. Finally, Section 5 concludes the paper.

\section{Computational model}

\subsection{Governing equations}

The two-dimensional reactive compressible flow simulations utilize the NS equations with the one-step two-species chemistry model as governing equations. Here, the simplified chemistry model is selected and fitted to physical parameters of a $\mathrm{H}_{2} / \mathrm{O}_{2}$ detonation. The details of the governing equations and the thermodynamic parameters of the chemistry model can be found in [17], while the reaction model is discussed in depth in [26]. At the end of the ZND reaction zone, the temperature is about $2500 \mathrm{~K}$ and the pressure is about $1.01325 \times 10^{5} \mathrm{~Pa}$ which gives the following transport parameters for the reaction model: $T_{r e f}=2500 \mathrm{~K}, \mu_{r e f}=1.07 \times 10^{-4} \mathrm{~Pa} \cdot \mathrm{s}, k_{r e f}=0.148 \mathrm{~W} /(\mathrm{m} \cdot \mathrm{K})$, $D_{\text {lref }}=5.5 \times 10^{-4} \mathrm{~m}^{2} / \mathrm{s}, D_{2 r e f}=6.4 \times 10^{-4} \mathrm{~m}^{2} / \mathrm{s}$. Matching the general trends and values at the end of the ZND reaction zone between the one-step and detailed reaction mode, the viscosity and conductivity values are eventually obtained from the Sutherland model, and the mass diffusion values are derived from a simple expression which includes the inverse dependence on pressure [17].

\subsection{Numerical method}

WENO schemes have been developed specifically to resolve both smooth regions and discontinuities. WENO schemes perform well for first-order hyperbolic problems, but they introduce plenty of numerical dissipation for second-order mixed equations with physical diffusion, which can 
pollute the diffusion effect. In contrast, no numerical viscosity is introduced for schemes developed with centered stencils; yet, numerical schemes based on centered stencils generally produce numerical oscillations at discontinuities, which can create instabilities in the simulations [24, 25].

Originally developed for large eddy simulations (LES) of turbulence, the hybrid WENO-CD scheme utilized here combines the advantages of both WENO and CD schemes. It is made up of two components: a finite-difference sixth-order WENO scheme to be used at discontinuities and a conservative sixth-order (CD) scheme for smooth-solution regions. The WENO stencil weights have been adjusted so that the optimal stencil matches the $\mathrm{CD}$ stencil around the matching boundaries. This can largely eliminate any dispersion errors resulting from switching between two schemes. The CD scheme imposes additional restrictions on the time integration. It is relatively easy to use explicit multistage time integration methods for the implementation with SAMR. Because of the stability properties of the explicit integration schemes, the preferred practical methods with the ability of inexpensive time adaptation in SAMR are Runge-Kutta method of third or higher order. Here the optimal third-order strong stability preserving (SSP) Runge-Kutta scheme [27] is used with a CFL parameter 0.99 in combination with time-splitting and the fourth-order accurate semi-implicit GRK4A method [28] for source term integration.

\subsection{Computational setup}

Here, detonation simulations are conducted in two-dimensional channels whose geometries are depicted in detail in Fig.1. A straight channel in Fig.1(a) is used in Section 3 while expanding channels in Fig.1(b) are studied in Section 4. 


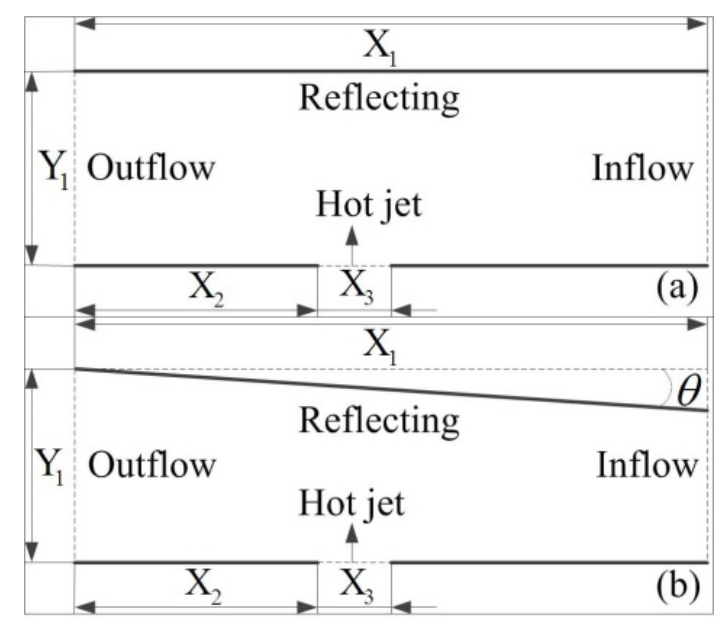

Fig.1 Schematics of computational setups.

The level-set technique [29] with ghost fluid approach [16] is employed for the expanding upper boundary in Fig.1(b). Reflecting boundary with slip wall conditions are used on the upper and lower wall, and the inflow of the hot jet is embedded within the lower domain boundary. In the viscid case, a turbulent boundary layer would develop along the inflow channel walls and its thickness will strongly depend on the length of the inflow section. Especially, at the start of the expansion section, the boundary layer will mostly detach, migrate partially into the channel interior and will only re-establish at the end of the expansion section. The process is rather complicate and can only accurately be described by simulating the entire combustion chamber. In order to eliminate this complexity and to be able to focus the present study just on the jet ignition process and immediately subsequent detonation propagation in the expansion section, we have opted to use the viscid model with slip boundary conditions in our investigations for not having to deal with boundary layers produced otherwise in the inflow section. Throughout the paper the supersonic flow of the reactive mixture is from right to left. The right boundary adopts the inflow condition and an ideal outflow condition is imposed on the left boundary. A mixture of $\mathrm{H}_{2} / \mathrm{O}_{2}$ detonation at $\mathrm{T}=300 \mathrm{~K}$ and $\mathrm{P}=6700 \mathrm{~Pa}$ flows at $\mathrm{V}_{\mathrm{CJ}}\left(\mathrm{V}_{\mathrm{CJ}}=1587.84 \mathrm{~m} / \mathrm{s}\right)$.

The inflow parameters of the hot jet are set to the values of the ZND state of a detonation in $\mathrm{H}_{2} / \mathrm{O}_{2}$ 
under the condition of the inflow pressure $6700 \mathrm{~Pa}$ and the temperature $300 \mathrm{~K}$. This gives the inflow parameters $\mathrm{P}=86376 \mathrm{~Pa}, \mathrm{~T}=1943.8 \mathrm{~K}, \rho=0.155 \mathrm{~kg} / \mathrm{m}^{3}$, Energy $349208 \mathrm{~J} / \mathrm{mol}$, mass fraction of $\mathrm{Y}_{1}$ $=0.0088$, mass fraction of $Y_{2}=0.9912$. The injection velocity is specified as the sonic speed to make it a chocked hot jet with velocity of $850 \mathrm{~m} / \mathrm{s}$.

\section{Results}

As shown in Fig.1(a), the length and height of the straight channel is $\mathrm{X}_{1}=75 \mathrm{~mm}$ and $\mathrm{Y}_{1}=25$ $\mathrm{mm}$, respectively. The distance between the hot jet and the head wall is $\mathrm{X}_{2}=25 \mathrm{~mm}$, and the width of the hot jet is $X_{3}=4 \mathrm{~mm}$. The initial base mesh is $600 \times 200$ cells. All computations are performed on a parallel cluster using 15 nodes, where each node has 20 cores. The processors are Intel E5-2692 (Ivy Bridge) with a clock speed of $2.2 \mathrm{GHz}$.

\subsection{Convergence analysis}

A series of simulations is conducted to investigate the effects of mesh resolution on the detonation evolution. The resolution parameters are given in Table 1.

Table 1 Mesh refinement parameters.

\begin{tabular}{ccccc}
\hline & Case 1 & Case 2 & Case 3 & Case 4 \\
\hline \multirow{3}{*}{ Method } & 4 levels & 5 levels & 6 levels & 7 levels \\
& $(2,2,2)$ & $(2,2,2,2)$ & $(2,2,2,2,2)$ & $(2,2,2,2,2,2)$ \\
& $\Delta_{\min }=1.56 \times 10^{-5} \mathrm{~m}$ & $\Delta_{\min }=7.8 \times 10^{-6} \mathrm{~m}$ & $\Delta_{\text {min }}=3.9 \times 10^{-6} \mathrm{~m}$ & $\Delta_{\min }=1.9 \times 10^{-6} \mathrm{~m}$ \\
\hline
\end{tabular}

Figure 2 depicts the simulation results of all cases at the same simulated physical time, which is a typical hot jet induced Mach reflection during the initiation process [10]. Overall, the same pattern of Mach stems, transverse waves, incident shocks and slip lines behind the triple points are presented in all the four cases, and especially these structures are all captured by the highest level of resolution. 


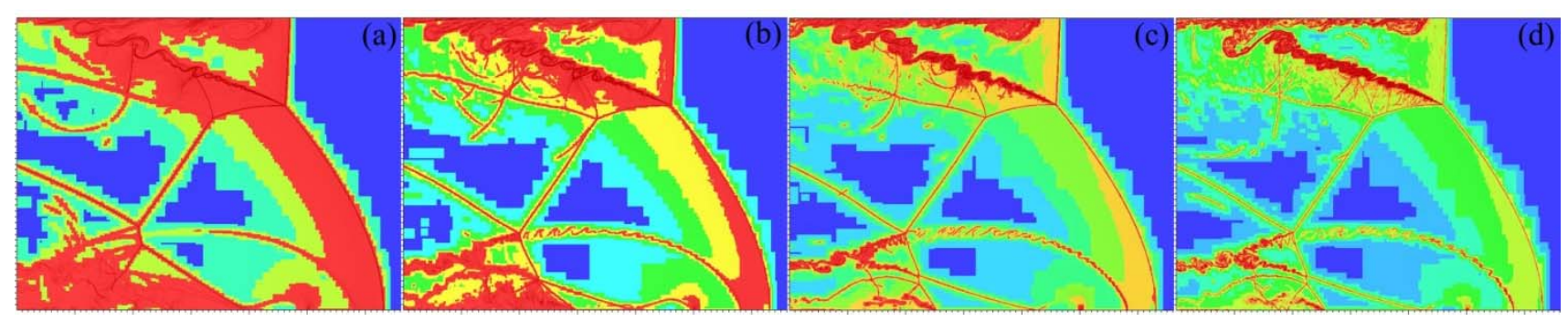

Fig.2 Detonation structure at $\mathrm{t}=140 \mu \mathrm{s}$ obtained using four mesh refinements given in Table (1), (a)

Refinement of Case 1, (b) Refinement of Case 2, (c) Refinement of Case 3, and (d) Refinement of

\section{Case 4.}

Before proceeding with the numerical simulations, it is worthwhile first to estimate the expected grid resolution, which is required to properly resolve diffusion effects. The diffusive scales are calculated using the average density $\left(0.589 \mathrm{~kg} / \mathrm{m}^{3}\right)$, temperature $(1373.5 \mathrm{~K})$ and pressure $\left(2.2 \times 10^{5} \mathrm{~Pa}\right)$ at the top and bottom of the shear layer behind the triple point near the upper boundary. The viscosity, mass diffusivity and thermal conductivity for these average parameters are $2.39 \times 10^{-5} \mathrm{~Pa} \cdot \mathrm{s}, 5.67 \times 10^{-5}$ $\mathrm{m}^{2} / \mathrm{s}$, and $3.31 \times 10^{-2} \mathrm{~W} /(\mathrm{m} \cdot \mathrm{K})$, respectively. Then the diffusive scales of viscosity $\left(\delta_{\text {visc }}=\sqrt{\frac{\mu t}{\rho}}\right)$, mass diffusivity $\left(\delta_{\text {mass }, 1}=\sqrt{\frac{D_{1} t}{\rho}}\right)$ and heat conductivity $\left(\delta_{\text {cond }}=\sqrt{\frac{k t}{\rho c_{v}}}\right)$ are evaluated as $7.54 \times 10^{-5} \mathrm{~m}$, $1.16 \times 10^{-4} \mathrm{~m}$ and $9.48 \times 10^{-5} \mathrm{~m}$, respectively, while the Reynolds number $\left(\operatorname{Re}=\sqrt{\frac{\rho a L}{\mu}}\right)$ is 1308 . Among the three scales, the viscous scale is the smallest, indicating that a fully resolved simulation should be limited by the viscous scale. As shown in Table 1, the highest mesh resolutions are all smaller than the viscous scale. Especially for Cases 2 to 4, at least about 10 to 40 cells are within the viscous scale, suggesting that these diffusive scales can be fully resolved except for the shock waves whose thicknesses are only several mean free path lengths. As a compromise between computational costs and numerical resolutions, five-level (Case 2 in Table 1) and six-level (Case 3 in Table 1) refinements have been used in the following simulations. 


\subsection{Scheme switching}

For the hybrid WENO-CD scheme, a switch is required so that regions of strong discontinuities are approximated by the WENO scheme, while within regions of smooth flow the CD scheme is used. Thus, we applied an improved scheme switching method based on a shock-based detection technique [30]. By utilizing an approximate Riemann solver, the method can both detect the strong shock waves and neglect weak ones, hence avoiding unrestricted usage of the WENO scheme. The structure of double Mach reflection (DMR) is formed near the upper boundary, as shown in Fig.2.

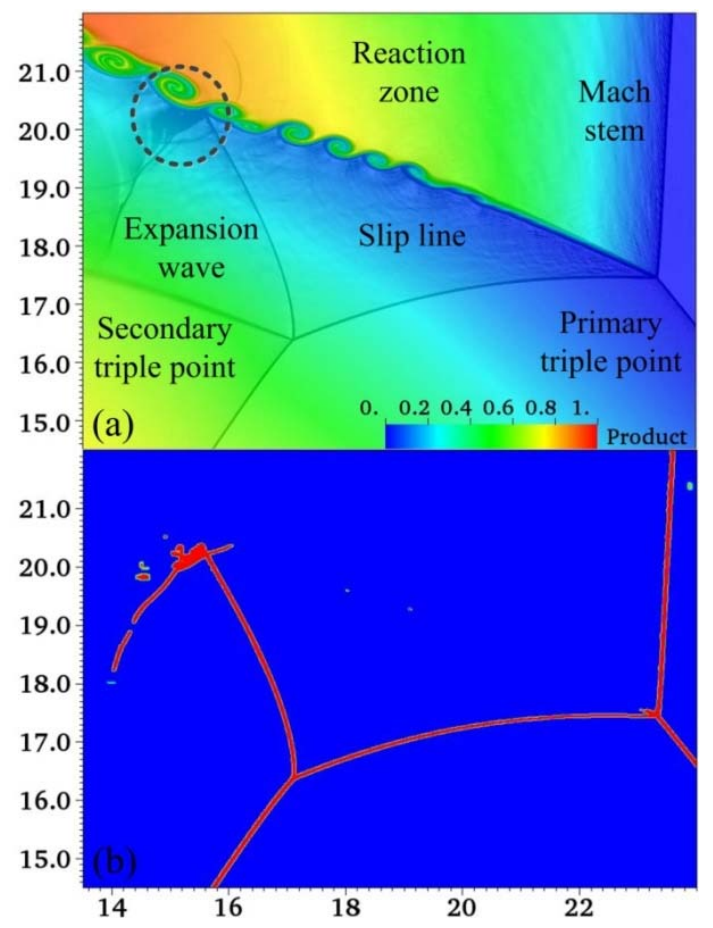

Fig.3 Double Mach reflection (DMR) structure for (a) Detailed structure of DMR and (b)

Distribution of WENO usage.

This is further evidenced in Fig.3(a) in which both the primary triple point and a secondary triple point are clearly visible. The Mach stem is coupled tightly with the reaction zone following behind, indicating that it is actually a Mach detonation. Directly behind the triple points are the shear layers and vortices resulted from $\mathrm{KH}$ instability. The expansion wave is also observed due to the reflection of the shock wave on the shear layer. As shown in Fig.3(b), the strong discontinuities around the 
primary triple point and the secondary triple point are all solved by the WENO scheme, while the other areas are solved by the CD scheme, and thus numerical dissipation is minimized to the extent possible.

\subsection{Viscosity effects}

The hybrid WENO-CD scheme is utilized both for viscous and inviscid detonations. Due to the high resolution and low dissipation of the hybrid scheme, diffusion effect in viscous detonations should be dominated by the physical viscosity, while numerical dissipation in inviscid detonations can be almost entirely avoided. It has been reported that the diffusion effect mainly occurs at shear layers and unburned mixture boundaries [22]. The comparison between viscous and inviscid detonations showed that the growth of secondary instabilities (KH instability type) is suppressed in NS simulations [31, 32]. In Fig.4, the shear layer, which is also the boundary of burned and unburned mixtures, is present in the DMR structure. It is seen that the DMR structures of NS and Euler simulations are qualitatively similar. The large-scale vortices resulting from RM instability underneath the upper wall behind the detonation front can also be observed. In Fig.4(a), the small-scale vortices resulting from $\mathrm{KH}$ instability along the shear layer are suppressed. In comparison, in Fig.4(b) irregular small-scale vortices are clearly visible both along the shear layer and underneath the upper wall. The results in Fig.4(b) are different from those in [17] where Euler equations are solved with a MUSCL-TVD scheme, which is due to the absence of not only physical viscosity in Euler equations but also numerical dissipation involved in the hybrid WENO-CD scheme. Compared with Fig.4(b) a more regular structure of the large-scale vortices can be observed in Fig.4(a). Nevertheless, the large-scale vortices both along the shear layers and underneath the upper wall have almost the same overall structures. Previous investigations indicate that the large-scale characteristics of a $\mathrm{KH}$ instability are visible when the shear layer is highly unstable [33] and the effect of viscosity and diffusion on the large-scale 
characteristics of a RM instability is negligible [34]. It may be concluded that diffusion effect can easily suppress the small-scale vortices produced by KH instability, but only play a minimal role in the suppression of the large-scale vortices associated with highly unstable shear layers and driven by RM instability.

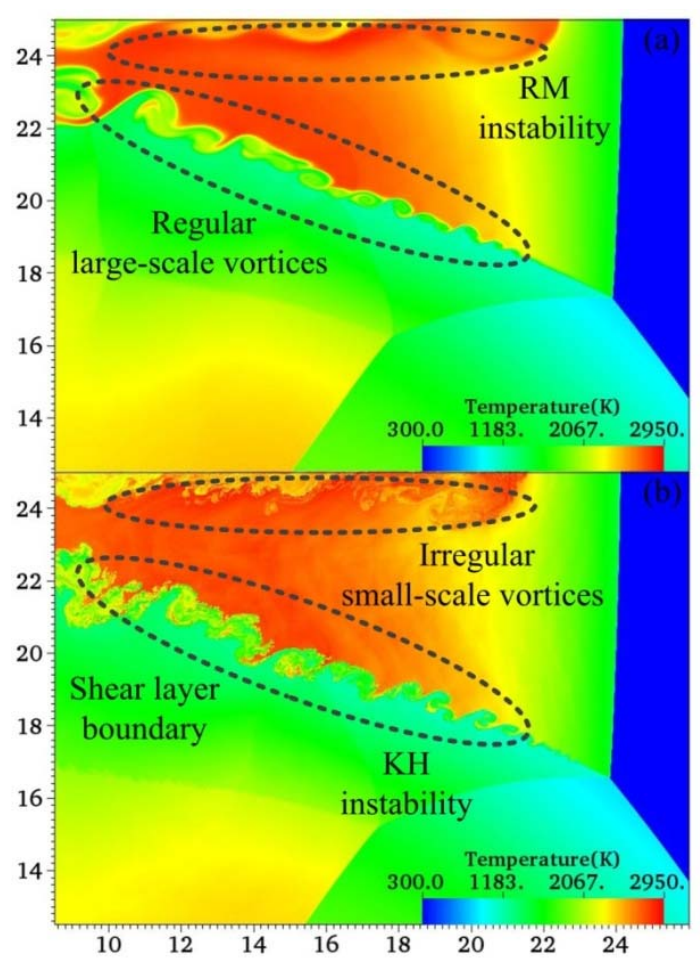

Fig.4 Comparison of the DMR structures, (a) NS solution and (b) Euler Solution.

\section{Detonation applications in expanding channels}

In this section, by solving the NS equations the detonation combustion in expanding channels is investigated for the application in scramjet combustors. Compared with the previously used straight channel, in this section we consider the initial expansion angle to be $\theta=3^{\circ}$ while all other simulation characteristics are identical, as shown in Fig.1(b). In this section we first examine the propagation of detonation after the injection and shutdown of the hot jet. Then we study the effect of expansion angle on the structure of detonation wave. Throughout this section the supersonic flow of the reactive mixture experiences a Prandtl-Meyer expansion with according flow acceleration in the expanding 
channel. When initiated, the detonation front is quasi-steady due to the inflow; yet, a slight continuous upstream propagation is noticed. Note that in contrast to the initiating jet, the inflow through the channel from the right never ceases.

\subsection{Detonation propagation after the injection of hot jet}

The structure of the detonation wave after the initiation of the hot jet is shown in Fig. 5. Figure 5(a)-(b) show the process of triple point collision on the upper wall together with the disappearance of an unburned jet and a detached shear layer. In a marginally stable detonation, the detonation front can ignite almost all the combustible mixtures through shock compression. However, due to the flow nonuniformity resulting from the expansion, an unburned jet occurs directly behind the expansion fan, as shown in Fig.5(a). Near the lower wall the triple point tends to propagate upward, while the detached shear layer is separated from the detonation front due to the triple point collision on the lower wall. As the triple point moves upward, the detached shear layer gradually vanishes and the unburned jet gradually becomes fully reacted because of the enhanced mixing between reactant and product as a result of diffusion effect, as shown in Fig.5(b). In contrast, Fig.5(c)-(d) show the triple point collision on the lower wall along with the formation of a new unburned jet and a detached shear layer. After the collision of the triple point with the upper wall in Fig.5(b), the flow behind the detonation front undergoes a RM instability due to the baroclinic vorticity production mechanism $[35,36]$, and results in generation of large-scale vortical structures. Subsequently a bifurcated detonation is formed resulting from the interaction of the jet flow with the Mach stem behind the expansion fan, as shown in Fig. 5(c). The large-scale vortical structures due to RM instability are clearly visible behind the detonation front. As the triple point moves downward, the unburned jet becomes elongated and partly burned, while the vortical structures gradually vanish. After the triple point collision with the lower 
wall in Fig.5(d), a new detached shear layer is formed. It is shown that for a stable detonation, detonation propagation with the hot jet injection is basically dominated by a laminar flow, where only a limited diffusion effect can occur when the shear layer interacts with the unburned jet flow. Results given in Fig.5 actually illustrate a fully periodic process of detonation propagation in an expanding channel from the new generation to the complete consumption of the unburned jet with an approximate period $\Delta \mathrm{T}_{1}=55 \mu \mathrm{s}$.

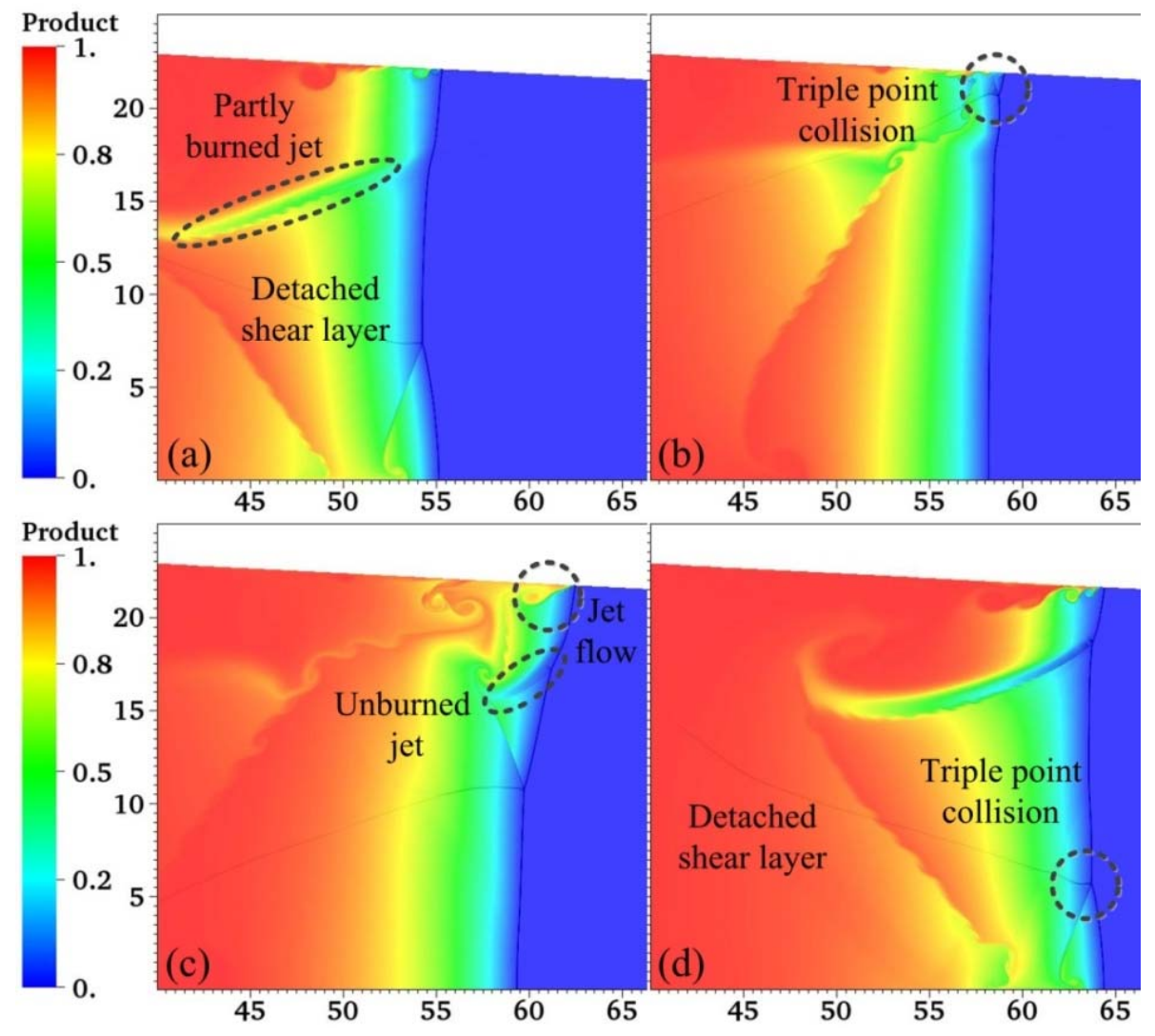

Fig.5 Detonation propagation in an expanding channel after the initiation of the hot jet, (a) $t=380$

$$
\mu \mathrm{s}, \text { (b) } \mathrm{t}=400 \mu \mathrm{s}, \text { (c) } \mathrm{t}=410 \mu \mathrm{s}, \text { (d) } \mathrm{t}=430 \mu \mathrm{s} \text {. }
$$

\subsection{Detonation propagation after the shutdown of hot jet}

Fig. 6 shows the continuous detonation propagation in the expanding channel when the hot jet has been shut down at $\mathrm{t}=350 \mu \mathrm{s}$. Even though a marginally stable detonation is adopted here, the 
detonation becomes more unstable and even turbulent compared with the situation of Fig.5. Fig.6(a)(b) show the growth of the unburned jet resulting from the expansion fan near the upper wall. In Fig.6(a), the unburned jet is initially formed and two triple points are about to collide. After the collision of the two triple points, large-scale vortices are generated by the RM instability. During this period, the unburned jet gradually grows longer, as shown in Fig.6(b). With its attenuation, the detonation front becomes weaker, thus resulting in more secondary triple points which can be inferred by comparing Fig.6(a) and Fig.6(c). In Fig.6(c), four secondary triple points coexist simultaneously thus generating more shear layers behind the triple points that undergo $\mathrm{KH}$ instability. Previous numerical and experimental investigations showed that in unstable detonations RM instability is the main mechanism for the generation of turbulent structures behind the detonation front [21], which indicated that the role of $\mathrm{KH}$ instability seems to be less important than that of the RM instability [22]. However, it is suggested here that the turbulent flow behind the detonation front is mainly induced by the vortex structures along the unburned jet, and that the highly unstable shear layers resulting from $\mathrm{KH}$ instability play a major role in the consumption of the unburned jet behind the expansion fan owing to the rapid turbulent mixing at the boundaries between shear layers and the unburned jet. After the unburned jet is fully burned, the released energy is increased and a stronger detonation is generated again, which results in the reduction of triple points and shear layers and the subsequent diffusion effect. As a result, a new unburned jet is formed as shown in Fig.6(d). Fig.6 also shows an approximately periodical process similar with that in Fig.5, but the period here is $\Delta \mathrm{T}_{2}=30 \mu \mathrm{s}$ which is only about half of $\Delta \mathrm{T}_{1}$ in Fig.5, indicating the importance of turbulent mixing compared with laminar mixing in the consumption of the unburned jet $[37,38]$. 


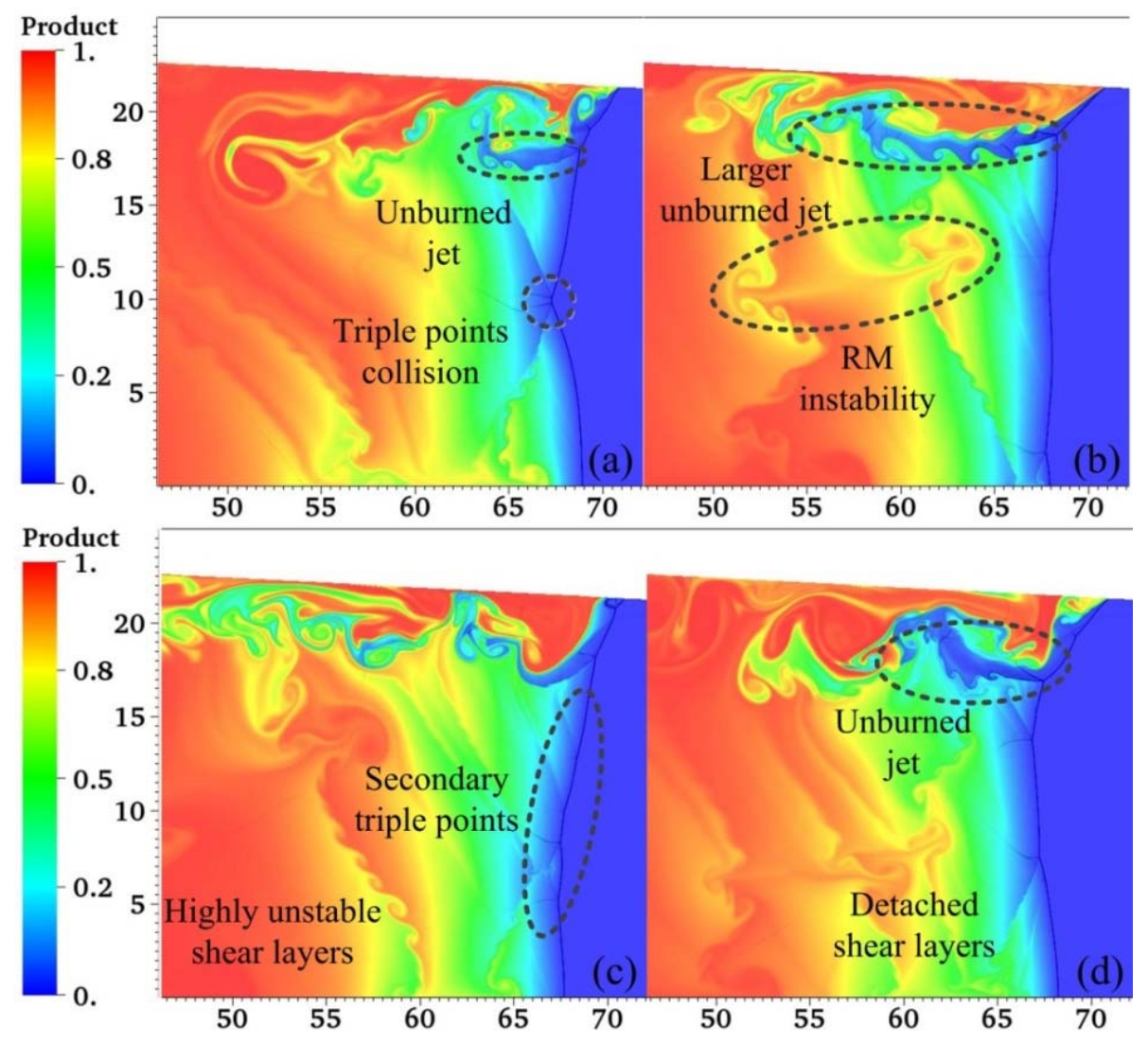

Fig.6 Propagation process in an expanding channel after the shutdown of the hot jet, (a) $t=595 \mu$,

$$
\text { (b) } \mathrm{t}=615 \mu \mathrm{s}, \text { (c) } \mathrm{t}=625 \mu \mathrm{s}, \text { (d) } \mathrm{t}=645 \mu \mathrm{s} \text {. }
$$

\subsection{Effects of the expansion angle}

As discussed in the previous sections, the expansion fan results in the non-uniformity of the supersonic combustible mixture, which subsequently plays an important role in detonation evolution in the expanding channel. Variations of the expansion angle change the corresponding expansion ratios and can influence on the characteristics of the expansion fan, hence leading to different non-uniform distributions of the unreacted mixtures.

Figure 7 shows schlieren images of density visualizing the detonation location shifting upstream as the expansion angle increases (i.e. $4^{\circ}, 5^{\circ}, 6^{\circ}$ ). This figure indicates that as the expansion angle increases the jet flow near the upper wall becomes more apparent and hence we see a large jet flow for 
the expansion angle of $6^{\circ}$. As the jet flow becomes larger, more vortices are created due to $\mathrm{KH}$ instability along the jet boundary, making the flow highly turbulent as can be seen by comparing Fig.7(a) and Fig.7(e). The generated turbulence can significantly enhance the mixing rate between burned products and partly unburned gases behind the leading shock and lead to the rapid consumption of the unburned reactants.

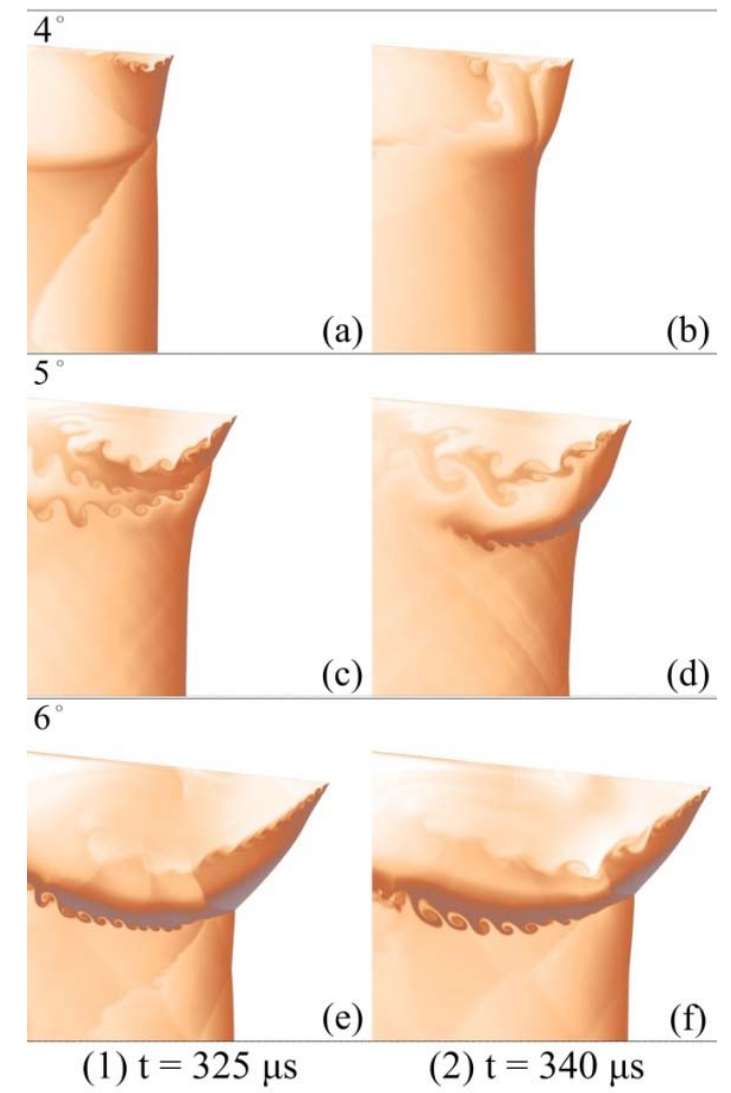

Fig.7 Detonation structures in channels with varying expansion angle.

Furthermore, with highly turbulent flow and with an adequate heat release from the rapid reactions of the unburned jet behind the expansion fan, detonations can propagate faster for larger expansion angles. Thus, at $\mathrm{t}=340 \mu \mathrm{s}$, the average positions of the detonation front for the three cases are $48 \mathrm{~mm}, 51.5 \mathrm{~mm}$ and $56 \mathrm{~mm}$, respectively. This indicates that as the expansion ratio increases along with the expansion angle, the size of the jet and the level of turbulence behind the detonation front increases. This results in rapid consumption of the unburned gases and hence the energy releases can support the self-sustained propagation of the detonation wave. Note that if the expanding angle is 
large enough, the detonation speed may not increase monotonously, and even detonation initiation might not be realizable anymore, which needs further study.

\section{Conclusion}

To investigate detonation combustion in supersonic combustible mixtures, the two-dimensional reactive NS equations with a one-step and two-species chemistry model are solved numerically using a sixth-order hybrid WENO-CD scheme with an adaptive mesh refinement method. The major results of the present work are as follows:

1. By comparing the results obtained by solving the NS and the Euler equations, it is found that in the inviscid simulation, small-scale vortices can be observed along the shear layers and large-scale vortical structures behind the detonation front. This behavior is due to the absence of both the physical viscosity in the Euler equations and thanks to the low numerical dissipation of the used hybrid scheme.

2. The physical diffusion effect inherent to the NS equations can suppress the small-scale vortices; however, it plays an almost negligible role in the evolution of large-scale vortices resulting from both the highly unstable $\mathrm{KH}$ instability along shear layers and RM instability behind the detonation front.

3. In the expanding channel, an unburned jet flow is generated behind the shock front because of the non-uniformity produced by the expansion fan. Due to the diffusion effect of detached shear layers, the unburned jet flow can be consumed through the mixing with the burned product. For a stable detonation, detonation propagation because of the hot jet injection is basically dominated by a laminar flow, where only a limited diffusion effect occurs when the shear layer interacts with the unburned jet flow. 
4. A highly turbulent flow is produced behind the detonation front after the shutdown of the hot jet owing to the formation of multiple secondary triple points and subsequent shear layers. Rather than the commonly known RM instability, large-scale vortices involved in highly unstable shear layers dominate the formation of the turbulent flow and the rapid turbulent mixing between unburned and burned mixture. Through the periodical formation and rapid consumption of the unburned jet, the detonation front can be maintained almost in the same position in the expanding channel.

5. Larger expansion angles can induce larger unburned jets and subsequently result in vortices due to $\mathrm{KH}$ instability of increasing size. The generated turbulence resulting from large-scale vortices significantly enhances the mixing rate between burned and unburned mixtures behind the Mach stem. Although the expansion ratio increases along with the expansion angle, the initiated detonations still propagate more quickly in channels with larger expansion angle.

\section{Acknowledgements}

This work is supported by National Natural Science Foundation of China (No. 91441201), Innovative Sustentation Fund for Excellent Ph.D. Students in NUDT (No. B140101) and Chinese Scholarship Council (CSC) (No. 201403170401).

\section{References}

[1] K. Kailasanath, AIAA J. 38(9) (2000) 1698-1708.

[2] B. Zhang, V. Kamenskihs, H.D. Ng, J.H.S. Lee, Proc. Combust. Inst. 33(2) (2011) 2265-2271.

[3] R. Knystautas, J.H.S. Lee, I.O. Moen, H.G. Wagner, Proc. Combust. Inst. 17(1979) 1235-1245. 
[4] K. Ishii, H. Kataoka, T. Kojima, Proc. Combust. Inst. 32(2009) 2323-2330.

[5] X. Han, J. Zhou, Z.Y. Lin, Chin. Phys. B. 21(2012) 124702.

[6] X. Han, J. Zhou, Z.Y. Lin, Y. Liu, Chin. Phys. Letters 30(2013) 054701.

[7] S.I. Jackson, J.E. Shepherd, AIAA J. 46(9) (2008) 2357-2367.

[8] J. Melguizo-Gavilanes, L. Bauwens, Int. J. Hydro. Energy 38(19) (2013) 8061-8067.

[9] Y. Lv, M. Ihme, Proc. Combust. Inst. 35 (2015) 1963-1972.

[10] X.D. Cai, J.H. Liang, Z.Y. Lin, R. Deiterding, H. Qin, X. Han, ASCE J. Aerosp. Eng. 28(1) (2015) 04014046.

[11] J.H. Liang, X.D. Cai, Z.Y. Lin, R. Deiterding, Acta Astronaut. 105(1) (2014) 265-277.

[12] X.D. Cai, J.H. Liang, Z.Y. Lin, R. Deiterding, Y. Liu, Aerosp. Sci. Tech. 39(2014) 442-455.

[13] X.D. Cai, J.H. Liang, Z.Y. Lin, R. Deiterding, F.C. Zhuang, Combust. Sci. Tech. 187(4) (2015) $525-536$.

[14] X.D. Cai, J.H. Liang, R. Deiterding, Y.G. Che, Z.Y. Lin, Int. J. Hydro. Energy 41(2016) 32223239

[15] Z. Liang, S. Browne, R. Deiterding, J.E. Shepherd, Proc. Combust. Inst. 31(2) (2007) 2445-2453.

[16] R. Deiterding, Comput. Struct. 87(11-12) (2009) 769-783.

[17] J.L. Ziegler, R. Deiterding, J.E. Shepherd, D.I. Pullin, J. Comput. Phys. 230(20) (2011) 7598-7630.

[18] M. Berger, J. Oliger, J. Comput. Phys. 53(3) (1984) 484-512.

[19] V.N. Gamezo, T. Ogawab, E.S. Oran, Proc. Combust. Inst. 31(2) 2007 2463-2471.

[20] V.N. Gamezo, T. Ogawab, E.S. Oran, Combust. Flame 155(1-2) 2008 302-315.

[21] K. Mazaheri, Y. Mahmoudi, M.I. Radulescu, Combust. Flame 159(6) (2012) 2138-2154.

[22] Y. Mahmoudi, N. Karimi, R. Deiterding, S.Emami, J. Propul. Power 30(2) (2014) 384-396.

[23] K.P. Grogan, M. Ihme, Proc. Combust. Inst. 35(2015) 2181-2189. 
[24] D.J. Hill, D.I. Pullin, J. Comput. Phys. 194(2004) 435-450.

[25] C. Pantano, R. Deiterding, D.J. Hill, D.I. Pullin. J. Comput. Phys. 221(2007) 63-87.

[26] S.P.M. Bane, J.L. Ziegler, J.E. Shepherd, Development of One-Step Chemistry Models for Flame and Ignition Simulation, Report No. GALCITFM: 2010.002, California Institute of Technology, 2010.

[27] S. Gottlieb, D.I. Ketcheson, C.W. Shu, J. Sci. Comput. 38(3) (2009) 251-289.

[28] P. Kaps, P. Rentrop, Numer. Math. 33(1979) 55-68.

[29] W. Mulder, S. Osher, J. Sethian, J. Comput. Phys. 100(2) (1992) 209-228.

[30] M. Lombardini, Richtmyer-Meshkov instability in converging geometries, $\mathrm{PhD}$ thesis, California Institute of Technology, California, USA, 2008.

[31] E.S. Oran, V.N. Gamezo, Combust. Flame 148(2007) 4-47.

[32] V.N. Gamezo, A.M. Khokhlov, E.S. Oran, Combust. Flame 126(2001) 1810-1826.

[33] F. Pingten, C.A. Eckett, J.M. Austin, J.E. Shepherd, Combust. Flame 133(2003) 211-229.

[34] M. Brouillette, Annu. Rev. Fluid Mech. 34(2002) 445-468.

[35] M.I. Radulescu, G.J. Sharpe, J.H.S. Lee, C.B. Kiyanda, A.J. Higgins, R.K. Hanson, Proc. Combust. Inst. 30(2) (2005) 1859-1867.

[36] G.J. Sharpe, J. Fluid Mech. 447(2001) 31-51.

[37] K. Mazaheri, Y. Mahmoudi, M. Sabzpooshani, M.I. Radulescu, Combust. Flame 162(2015) 26382659.

[38] Y. Mahmoudi, K. Mazaheri, Acta Astronaut. 115(2015) 40-51. 


\section{List of figure captions:}

Fig.1 Schematics of computational setups.

Fig.2 Detonation structure at $\mathrm{t}=140 \mu \mathrm{s}$ obtained using four mesh refinements given in Table (1), (a)

Refinement of Case 1, (b) Refinement of Case 2, (c) Refinement of Case 3, and (d) Refinement of Case 4.

Fig.3 Double Mach reflection (DMR) structure for (a) Detailed structure of DMR and (b) Distribution of WENO usage.

Fig.4 Comparison of the DMR structures, (a) NS solution and (b) Euler Solution.

Fig.5 Detonation propagation in an expanding channel after the injection of hot jet, (a) $t=380 \mu \mathrm{s}$, (b) $\mathrm{t}=400 \mu \mathrm{s},(\mathrm{c}) \mathrm{t}=410 \mu \mathrm{s},(\mathrm{d}) \mathrm{t}=430 \mu \mathrm{s}$.

Fig.6 Propagation process in an expanding channel after the shutdown of the hot jet, (a) $t=595 \mu$, (b) $\mathrm{t}=615 \mu \mathrm{s}$, (c) $\mathrm{t}=625 \mu \mathrm{s}$, (d) $\mathrm{t}=645 \mu \mathrm{s}$.

Fig.7 Detonation structures in channels with varying expansion angle. 